\title{
A simple method to determine charge-dependent curvature biases in track reconstruction in hadron collider experiments
}

\author{
William Barter ${ }^{1, \mathrm{a}}$, Martina Pili ${ }^{2, \mathrm{~b}}$, Mika Vesterinen ${ }^{3, \mathrm{c}}$ \\ ${ }^{1}$ Imperial College London, London, UK \\ ${ }^{2}$ University of Oxford, Oxford, UK \\ ${ }^{3}$ University of Warwick, Coventry, UK
}

Received: 15 January 2021 / Accepted: 27 February 2021 / Published online: 22 March 2021

(C) The Author(s) 2021

\begin{abstract}
A new data-driven method, using $Z \rightarrow \mu^{+} \mu^{-}$ decays, is proposed to correct for charge-dependent curvature biases in spectrometer experiments at hadron colliders. The method is studied assuming a detector with a "forwardspectrometer" geometry similar to that of the LHCb experiment, and is shown to reliably control several simplified detector mis-alignment configurations. The applicability of the method for use in measurements of precision electroweak observables is evaluated.
\end{abstract}

\section{Introduction}

The measurement of charged particle momenta in hadron collider experiments is susceptible to mis-alignments, inaccuracies in the knowledge of the magnetic field, or other biases in the reconstruction algorithms. Precision measurements of electroweak parameters such as the $W$ boson mass $\left(m_{W}\right)$ and the weak mixing angle $\left(\sin ^{2} \theta_{\text {lept }}^{\text {eff }}\right)$, using muonic decays of weak bosons, are particularly sensitive to the accuracy with which these details are modelled in simulations of the signal processes. In this paper we focus on curvature biases of the type

$\frac{q}{p} \rightarrow \frac{q}{p}+\delta$,

for particles of charge $q$ and momentum $p$ driven, for example, by weak modes in the detector alignment procedure. It is desirable to identify and eliminate these $(\delta)$ biases from both the real and simulated data, so that the simulation can be tuned and validated with better reliability.

\footnotetext{
a e-mail: william.barter@cern.ch

b e-mail: martina.pili@cern.ch (corresponding author)

ce-mail: mika.vesterinen@cern.ch
}

In the first measurement of $m_{W}$ with the ATLAS experiment [1], track curvature biases were determined using the ratio of the energy and momentum of electrons from $W \rightarrow e v$ decays. This approach is only applicable for detectors with comparable kinematic resolution for electrons and muons. A second approach, based on the determination of sagitta biases in $Z \rightarrow \mu^{+} \mu^{-}$decays was also studied. The dependence of the invariant mass of $Z \rightarrow \mu^{+} \mu^{-}$decays on the muon kinematics is sensitive to curvature biases but, particularly in the case of global mis-alignments, the biases on the $\mu^{+}$and $\mu^{-}$momenta can be strongly anti-correlated. Ref. [2] details a method in which the curvature biases are determined by assuming that the mean $1 / p_{T}$ of the $\mu^{+}$and $\mu^{-}$should follow the expectation of simulation, where $p_{T}$ is the momentum of the muon transverse to the beam axis. The reliance on simulation arises because the $p_{T}$ distributions are sensitive to both mis-alignment and physics effects (including intrinsic differences between the $\mu^{+}$and $\mu^{-}$kinematic distributions in $Z$ decays). That method has nevertheless been successfully applied in, for example, measurements of $\sin ^{2} \theta_{\text {lept }}^{\text {eff }}$ by CDF [3] and CMS [4].

This paper presents an alternative data-driven approach to determine charge-dependent curvature bias corrections using $Z \rightarrow \mu^{+} \mu^{-}$decays. The method is validated with the example of the LHCb experiment [5] using a simplified model of the detector geometry and mis-alignment configurations.

\section{The simulated event sample}

A sample of $10^{8} p p \rightarrow Z \rightarrow \mu^{+} \mu^{-}$events, at a centreof-mass-energy of $13 \mathrm{TeV}$, is generated with POWHEG Box [6,7]. These events are subsequently processed with Pythia8 [8], which simulates a QCD parton shower, hadronisation, the underlying event, and QED final state radiation. Momentum resolution smearing is considered to be unnec- 
essary for the present study, which is focused on systematic curvature biases rather than the intrinsic curvature resolution. Since this work was conducted in the context of precision measurements of electroweak observables with $\mathrm{LHCb}$ [913 ], events are selected with both muons in the pseudorapidity interval $1.7<\eta<5$. It is also required that both muons have $p_{T}>15 \mathrm{GeV}$ and that at least one of the two muons has $p_{T}>25 \mathrm{GeV}$. The coordinates are fixed according to a righthanded Cartesian system with the origin at the $p p$ interaction point. The $x$-axis is oriented horizontally towards the outside of the LHC ring, the $y$-axis points upwards with respect to the beamline and the $z$-axis is aligned with the beam direction. The simplified geometry includes a dipole magnetic field along $y$, bending the tracks on the $x-z$ plane and a single measurement plane after the magnet. Charged particles are deflected along the $x$ axis by $p^{-1} \times 3 \mathrm{GeV}$, which roughly corresponds to the bending power of the $\mathrm{LHCb}$ spectrometer [5]. A curvature bias $(\delta)$ can be interpreted as a systematic translation of the measurement plane along the $x$ axis:

$\Delta x=\delta \times 3 \mathrm{GeVm}$

In Sect. 6 it is estimated that a $\Delta x$ value of $5 \mu \mathrm{m}$ leads to a bias of $\mathcal{O}(50) \mathrm{MeV}$ in the determination of $m_{W}$ for a single charge. It should be noted that the bias in the measurement of $m_{W}$ for a single charge is strongly (but not fully) anticorrelated to the corresponding bias in $m_{W}$ for the opposite charge, leading to partial cancellations when the combination of the two charges is considered. Nevertheless, the precision of the measurement is potentially affected by such biases. Given that, for example, the LHCb Run-II dataset permits an $\mathcal{O}(10) \mathrm{MeV}$ statistical precision on $m_{W}$ [9] a simple and reliable method to control this source of bias is required.

\section{The pseudomass method}

The proposed method relies on an approximation of the invariant mass of $Z \rightarrow \mu^{+} \mu^{-}$decays using the momentum of one muon and only the direction of the other. In the context of a measurement of the differential cross section for $p \bar{p} \rightarrow Z \rightarrow \mu^{+} \mu^{-}$with the D0 experiment, Ref. [14] introduced the pseudomass ${ }^{1}\left(M^{ \pm}\right)$for each muon charge as:

$$
M^{ \pm}=\sqrt{2 p^{ \pm} p_{T}^{ \pm} \frac{p^{\mp}}{p_{T}^{\mp}}(1-\cos \theta)},
$$

\footnotetext{
1 The definition of "pseudomass" in D0 is given in terms of leading/subleading muons, not positive/negatively charged muons. Furthermore, the pseudomass was used for different purposes, and not for addressing curvature biases.
}

where $p^{ \pm}$and $p_{T}^{ \pm}$are the momenta and transverse momenta of the $\mu^{ \pm}$and $\theta$ is the opening angle between the two muons. The pseudomass is an estimate of the dimuon mass under the assumption that the dimuon system has zero momentum transverse to the bisector of the two lepton transverse momenta. ${ }^{2}$ This assumption is inspired by the fact that most of the $Z \rightarrow \mu^{+} \mu^{-}$cross section at hadron colliders is in the region $p_{T}^{Z}<m_{Z}$. A subset of events with smaller $p_{T}^{Z}$ values can be selected, independently of the muon momenta, using the $\phi^{*}$ variable [15], defined as:

$\phi^{*} \equiv \tan \left(\phi_{\mathrm{acop}} / 2\right) \sin \theta_{\eta}^{*} \approx \frac{p_{T}^{Z}}{m_{Z}}$,

where $\phi_{\text {acop }}=(\pi-\Delta \phi)$ and $\Delta \phi$ is the azimuthal opening angle between the two leptons and $\cos \theta_{\eta}^{*} \equiv \tanh \left(\frac{\eta^{-}-\eta^{+}}{2}\right)$, and where $\eta^{-}$and $\eta^{+}$are the pseudorapidities of the negatively and positively charged leptons, respectively. A requirement of $\phi^{*}<0.05$ corresponds to roughly half of the available events. Figure 1 (left) shows how this requirement selects events with smaller $p_{T}^{Z}$ on average, while Fig. 1 (right) shows that the pseudomass distribution of these events has a narrow width of $\mathcal{O}(5) \mathrm{GeV}$.

Figure 2 shows that the peaks of the $M^{+}$and $M^{-}$distributions are displaced by around $2 \mathrm{GeV}$ when $\Delta x=50 \mu \mathrm{m}$ is assumed in the simulation. This sensitivity can be exploited to determine the $\delta$ in Eq. 1 through a simultaneous likelihood fit of the $M^{+}$and $M^{-}$distributions. Both distributions are modelled by a double Crystal Ball (CB) function [16], plus an exponential function for the non-peaking component of the $Z / \gamma^{*}$ line-shape. The mean of the CB function for the $M^{ \pm}$ distribution is defined as $\bar{M}(1 \pm A)$, where $\bar{M}$ and the asymmetry $A$ are two of the 16 floating parameters of the fit. The other floating parameters are the width of the first $\mathrm{CB}$ function and the relative width of the second $\mathrm{CB}$ (for both $M^{+}$and $M^{-}$); the $\alpha, n$ parameters describing the tail of the $\mathrm{CB}$; the slope of the exponential component; the normalisation constants of the three shape functions. The charge-dependent curvature bias can be determined via

$\delta \approx A \frac{\left\langle\frac{1}{p^{+}}\right\rangle+\left\langle\frac{1}{p^{-}}\right\rangle}{2}$.

Since this approach decouples the effect of curvature biases on the momenta of the $\mu^{+}$and $\mu^{-}$it is straightforward to determine $\delta$ values in an arbitrary number of bins of $\eta$ and $\phi$. The curvature biases can be determined independently in each bin. Unless otherwise specified a binning scheme with 8 (12) $\eta(\phi)$ bins is used in the remainder of this paper.

It is anticipated that the determination of the $\delta$ values with Eq. 5 will be slightly biased by the forward-backward asym-

\footnotetext{
${ }^{2}$ Ref. [15] refers to this axis as $\hat{b}$.
} 

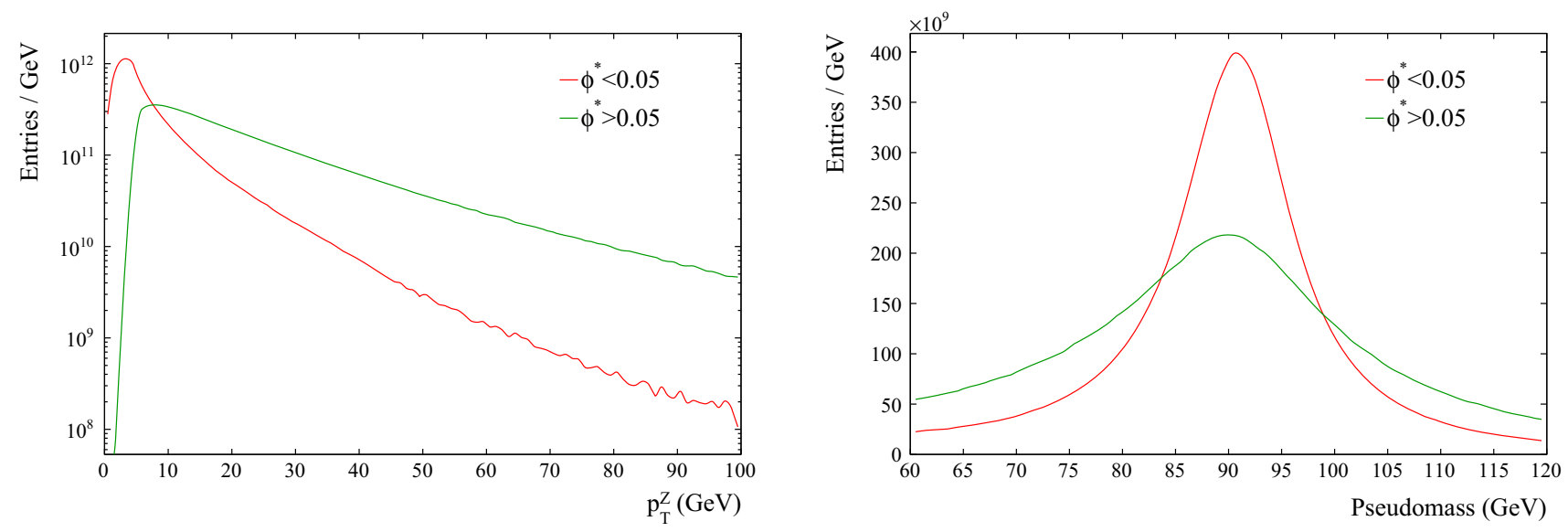

Fig. 1 The distribution of $p_{T}^{Z}$ (left) and the pseudomass (right) for events passing and failing the $\phi^{*}<0.05$ cut

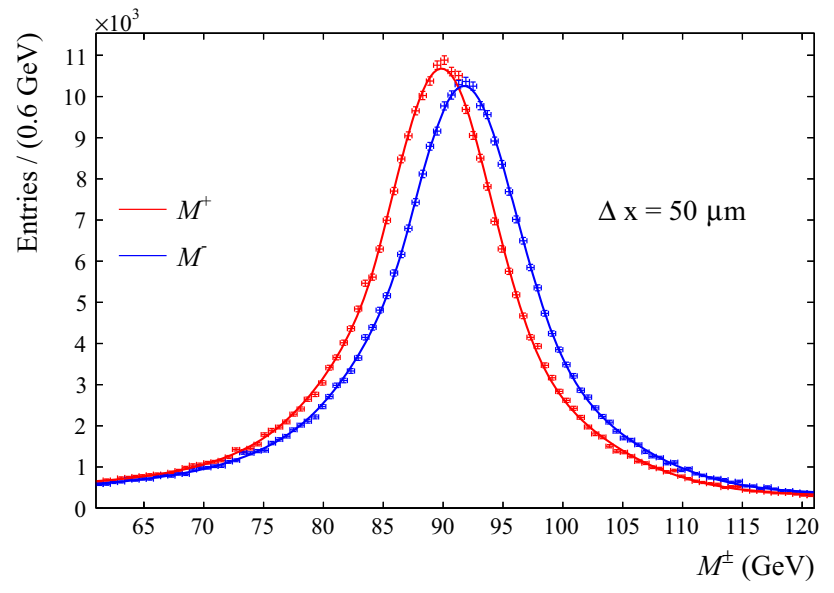

Fig. 2 The parametric fit of the $M^{+}$and $M^{-}$pseudomass distributions, in the presence of a $\Delta x=50 \mu \mathrm{m}$. The signal is modelled with a double Crystal Ball function, while the non-peaking component of the $Z / \gamma^{*}$ line-shape is modelled with an exponential

metry $\left(A_{F B}\right)$ in the $Z \rightarrow \mu^{+} \mu^{-}$process, which implies a small difference in the kinematic distributions of the $\mu^{+}$and $\mu^{-}$. However, Eq. 5 relies on the asymmetry between the pseudomass peak positions and $A_{F B}$ is minimal for dimuon masses close to the peak of the $Z$ resonance. This bias is nevertheless evaluated and addressed in the following section.

\section{Validation of the method}

The pseudomass method is validated with 30 toy experiments using statistically independent subsets of the $Z \rightarrow \mu^{+} \mu^{-}$ event sample described in Sect. 2. When no mis-alignment is simulated the method should return $\Delta x$ values that are statistically compatible with zero.

Figure 3 (upper row) shows the distribution over the 30 toys of the pull, i.e. the difference between the correc- tion derived using the proposed method and the expectation $(\Delta x=0 \mu \mathrm{m})$, divided by the statistical uncertainty of the measured parameter in each of the $\eta$ bins. In this study an integration over $\phi$ bins is performed. Given the forwardbackward asymmetry it is not surprising to see that the pull distributions have means that are systematically shifted from zero, by up to three standard deviations, with a strong dependence on $\eta$. Figure 3 (central row) shows that this bias is eliminated when the charges of the $Z$ boson decay products are randomised, so that the number of $\mu^{+}$and $\mu^{-}$falling in each $[\eta, \phi]$ bin is the same (i.e. effectively "switching off" the forward-backward asymmetry). The small bias can be corrected using simulation but, given the intended application of the method to measurements of $m_{W}$ and $\sin ^{2} \theta_{\text {lept }}^{\text {eff }}$, careful attention is required for the dependence of this bias on the value of $\sin ^{2} \theta_{\text {lept }}^{\text {eff }}$ assumed in the simulation. A larger (than in the 30 toy experiments) sample of around $10^{7}$ events is used to determine this correction with high statistical precision. Figure 4 (left) shows the resulting pseudomass asymmetry values in bins of $\eta$ for the nominal value of $\sin ^{2} \theta_{\text {lept }}^{\text {eff }}$ (black points) and $\pm 5 \times 10^{-2}$ variations (red and blue points). It is reassuring to see that the pseudomass asymmetry shifts by at most $5 \times 10^{-4}$ for these extreme variations, corresponding to roughly \pm 300 times the uncertainty on the current world average value of $\sin ^{2} \theta_{\text {lept }}^{\text {eff }}$ [17]. Figure 4 (right) shows that the same variations in $\sin ^{2} \theta_{\text {lept }}^{\text {eff }}$ lead to far greater changes in the profile of the mean $1 / p_{T}$ asymmetry versus $\eta$. The pseudomass asymmetry therefore appears to be better suited than the $1 / p_{T}$ asymmetry for the determination of curvature biases with $Z \rightarrow \mu^{+} \mu^{-}$events. A small correction for this bias can now be included in Eq. 5, as follows:

$\delta \approx\left(A-A_{F B, \mathrm{corr}}\right) \frac{\left\langle\frac{1}{p^{+}}\right\rangle+\left\langle\frac{1}{p^{-}}\right\rangle}{2}$, 
where $A_{F B \text {,corr }}$ corresponds to the black points in Fig. 4 . Figure 3 (lower row) shows that using Eq. 6 the curvature biases can be determined without bias, since the pull distributions of the $\Delta x$ values are consistent with standard normal distributions in all the bins considered.

\section{Application of the method}

The pseudomass method is tested on a subset of $3 \times 10^{6}$ events from the sample described in Sect. 2. The muon momenta are re-calculated to mimic the effects of five mis-alignment scenarios that are representative of the LHCb detector [18]. There is a distinction between coherent translations/rotations of the entire measurement plane and incoherent translations in bins of $\eta$ and $\phi$. In all cases the additional deflection $(\Delta x)$ of a track along $x$ due to the introduced mis-alignment is calculated, and the corresponding variation in the momentum is derived from Eq. 2. The five mis-alignment scenarios are configured as follows.

1. Coherent translation along $x(\Delta x=50 \mu \mathrm{m})$.

2. Coherent translation along $z(\Delta z=100 \mu \mathrm{m})$ : the corresponding deflection along $x$ is

$$
\Delta x=\Delta z \frac{\cos \phi}{\sinh \eta} .
$$

3. Coherent rotation in the $x-y$ plane $\left(R_{z}=0.2 \mathrm{mrad}\right)$ : the corresponding deflection along $x$ is

$$
\Delta x=\frac{\cos \left(\phi+R_{z}\right)-\cos \phi}{\cos \phi} \frac{\mathrm{m}}{p / \mathrm{GeV}} .
$$

4. Incoherent translation along $x$, with the $\Delta x$ values randomly sampled from a symmetric Gaussian distribution with a width of $100 \mu \mathrm{m}$ in each of the $\eta$ and $\phi$ bins corresponding to the same binning scheme as the pseudomass corrections.

5. Incoherent translation along $x$ with five $\eta$ bins instead of eight, so that the binning scheme is slightly different to that used in the pseudomass corrections.

Maps of the curvature bias corrections in the $\eta$ and $\phi$ bins are determined for each mis-alignment scenario, and they are presented in Fig. 5. An iterative procedure is required because Eq. 5 is only accurate to leading order. The residual corrections of each iterations are added to the corrections map of the previous iteration. Iterations stop when the size of the residual corrections are zero within their statistical errors in most $[\eta, \phi]$ bins. This happens with around two iterations. Figure 6 shows the dimuon invariant mass $\left(M_{\mu \mu}\right)$ distribution and the forward backward asymmetry in bins of $M_{\mu \mu}$ in the simulation. The black histogram and points correspond to the simulated events before mis-alignment, while the magenta (green) versions correspond to the misaligned (coherent $\Delta x=50 \mu \mathrm{m}$ scenario) simulation before (after) the pseudomass corrections. It can be seen that the mis-alignment degrades the $M_{\mu \mu}$ resolution by around $10 \%$ and biases the $A_{F B}$ values by up to $30 \%$ in some mass bins. The pseudomass method successfully restores the original mass resolution and $A_{F B}$ profile. Figure 7 shows the profiles of the mean $M_{\mu \mu}$ as a function of the $\phi$ of the $\mu^{+}$and $\mu^{-}$ and as a function of the angle $\phi_{d}$ between decay plane normal and the magnetic field direction. In the simulated LHCb-like geometry $\phi_{d}$ distinguishes tracks with opposite-sign curvature in the $x-z$ plane. The lower row of Fig. 7 shows the values of $A_{F B}$ as a function of $M_{\mu \mu}$. The left (right) columns correspond to before (after) applying the pseudomass corrections. The black points correspond to the simulation before mis-alignment while the other three colours correspond to the first three mis-alignment scenarios. It can be seen that the profile of $A_{F B}$ versus $M_{\mu \mu}$ is particularly sensitive to the $\Delta x$ translation, while the $\phi_{ \pm}$and $\phi_{d}$ profiles are sensitive to the $\Delta z$ translation. The pseudomass corrections reliably resolve both of these pathologies, as can be seen in the righthand column of Fig. 7.

Figure 8 shows the same four alignment-sensitive profiles as in Fig. 7 but considering incoherent mis-alignments along the $x$ axis. By construction, the size of the local mis-alignment depends on random numbers. Therefore, it is appropriate to repeat the study for multiple toy data sets, generated sampling the mis-alignments from different random seeds. The bands in Fig. 8 are centered on the mean value across 10 toys, and their width is given by their root mean square (RMS). The red (blue) bands correspond to the values before (after) applying the pseudomass corrections to the mis-aligned events. For most points the blue bands are at least a factor of two narrower than the red bands. The reduction in the width of the band is particularly evident for the mass dependence of $A_{F B}$. Similar results are obtained for the fifth mis-alignment scenario, corresponding to a different binning scheme in $\eta$ and $\phi$.

It is now interesting to test the susceptibility of measurements of $m_{W}$ and $\sin ^{2} \theta_{\text {lept }}^{\text {eff }}$ to these mis-alignment scenarios. For each of the first four mis-alignment scenarios, values of $m_{W^{+}}, m_{W^{-}}$and $\sin ^{2} \theta_{\text {lept }}^{\text {eff }}$ are extracted from the same sample of generator level events, using a simple single-parameter $\chi^{2}$ fit described in greater detail in Sect. 6. With the assumed signal yields for LHCb Run-II [10], the statistical uncertainties on $m_{W^{+}}, m_{W^{-}}$, and $\sin ^{2} \theta_{\text {lept }}^{\text {eff }}$ are $9 \mathrm{MeV}, 11 \mathrm{MeV}$ and $43 \times 10^{-5}$, respectively. Table 1 lists, for each of the three parameters of interest, the shift in the measured value and the $\Delta \chi^{2}$ that is induced by the first four mis-alignment scenarios. The overall bias for $m_{W}$ is obtained from the weighted 

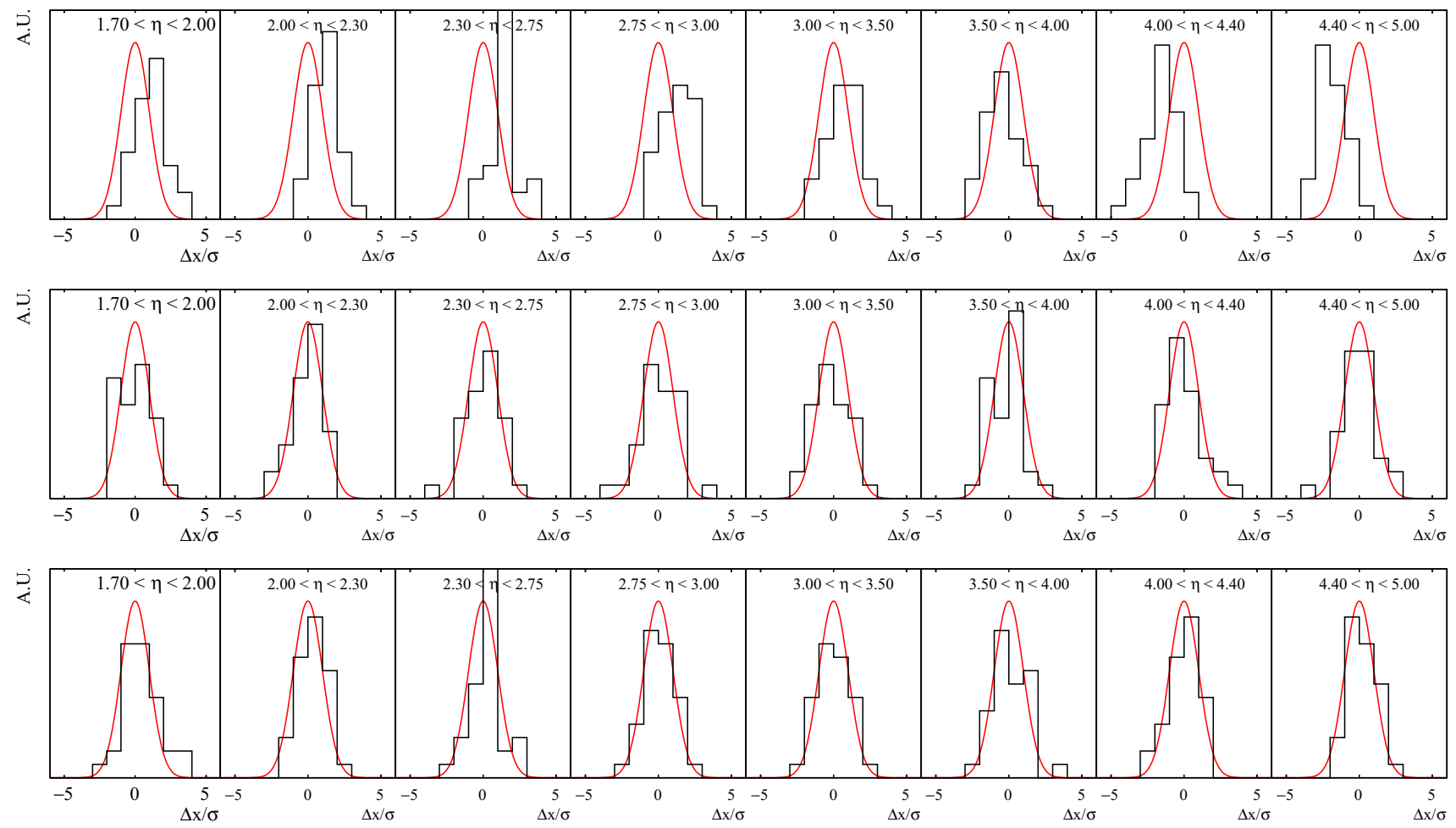

Fig. 3 The distribution of the difference between the measured $\Delta x$ values calculated with the pseudomass method and the expectation $(\Delta x=0 \mu \mathrm{m})$ divided by the uncertainty of the measured parameter in bins of $\eta$ for 30 toy experiments. The upper two rows correspond to Eq. 5. In the central row the muon charges have been randomised. In the lower row Eq. 6 is used
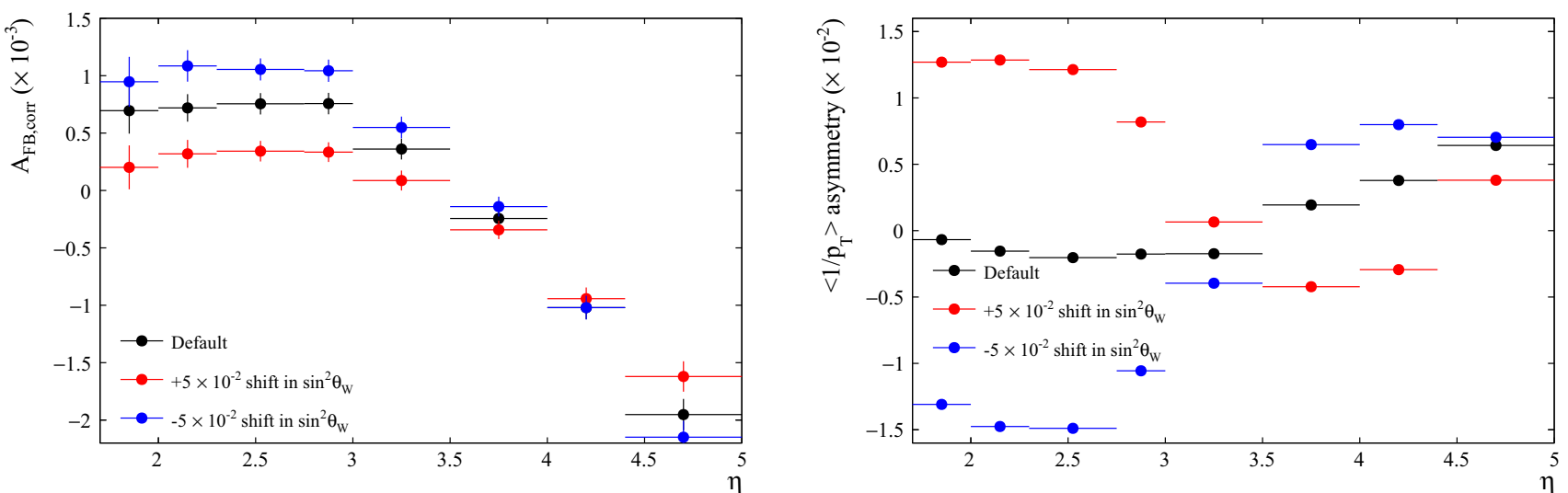

Fig. 4 The pseudomass (left) asymmetry and muon $1 / p_{T}$ (right) asymmetry in bins of the muon pseudorapidity. The asymmetries are shown for three different values of $\sin ^{2} \theta_{\text {lept }}^{\text {eff }}$, including two extreme variations (red and blue) around the nominal value (black)

average of the biases for $m_{W^{+}}$and $m_{W^{-}}$, and is reported in a separate column in Table 1 . The largest biases in all three parameters are caused by the (coherent and incoherent) mis-alignments along the $x$ direction. Rotations around, or translations along, the $z$ axis lead to smaller biases. The next obvious step is to understand whether the pseudomass method is able to correct for such biases.

\section{Impact of the pseudomass method on the measurement of electroweak observables}

The sample of $Z \rightarrow \mu^{+} \mu^{-}$events described in Sect. 2 is complemented by samples of $2 \times 10^{7} W^{-} \rightarrow \mu^{-} \bar{v}_{\mu}$ decays and $2 \times 10^{7} \mathrm{~W}^{+} \rightarrow \mu^{+} v_{\mu}$ decays. Events are selected with muons in the region $2<|\eta|<5$, reducing both samples to around $10^{7}$ events. For each of the three parameters of 

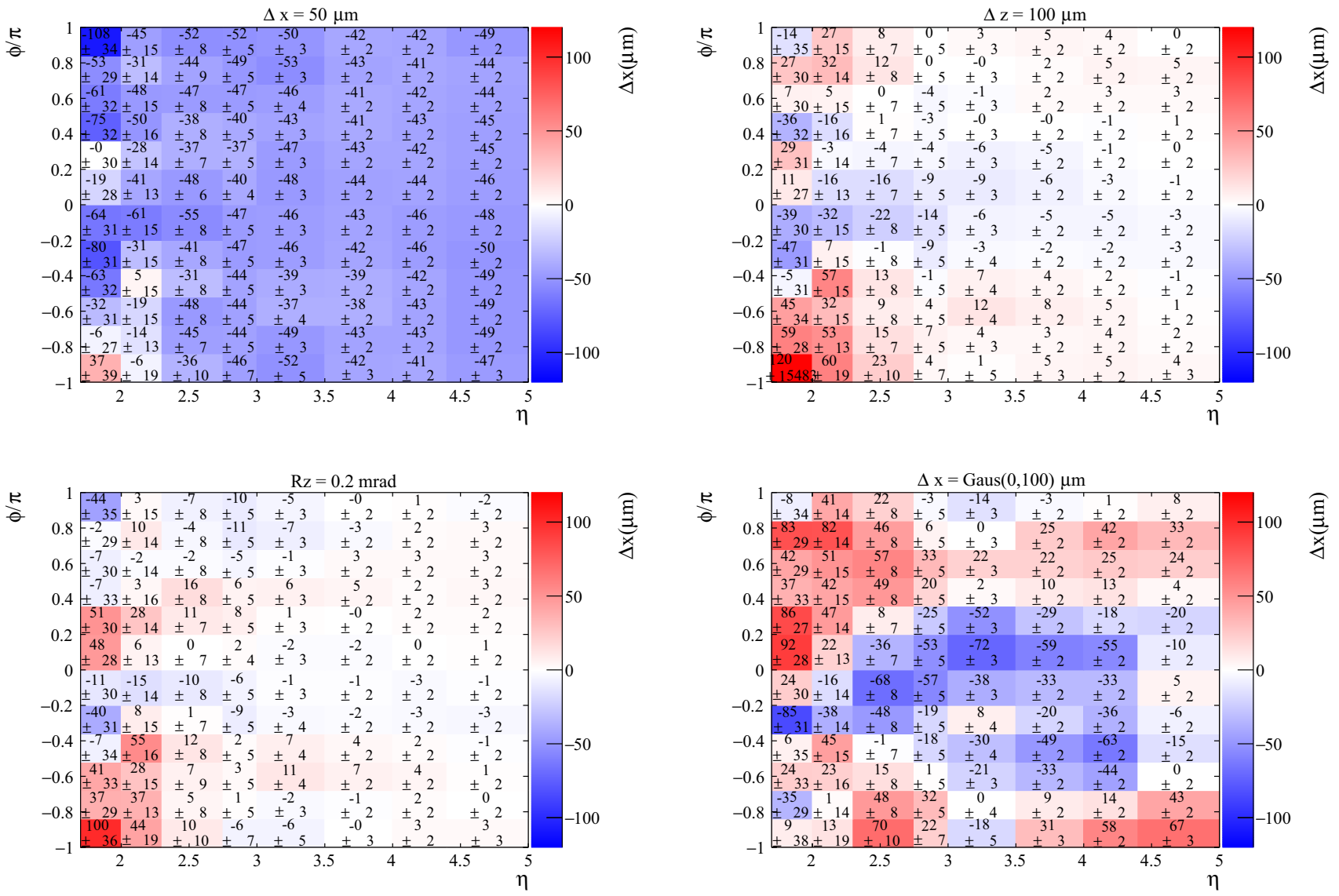

Fig. 5 The pseudomass correction in bins of $[\eta, \phi]$ for (clockwise from upper left) a coherent $\Delta x=50 \mu \mathrm{m}$, coherent $\Delta z=100 \mu \mathrm{m}$, incoherent $\Delta x=\operatorname{Gaus}(0,100) \mu \mathrm{m}$, coherent $R_{z}=0.2 \mathrm{mrad}$

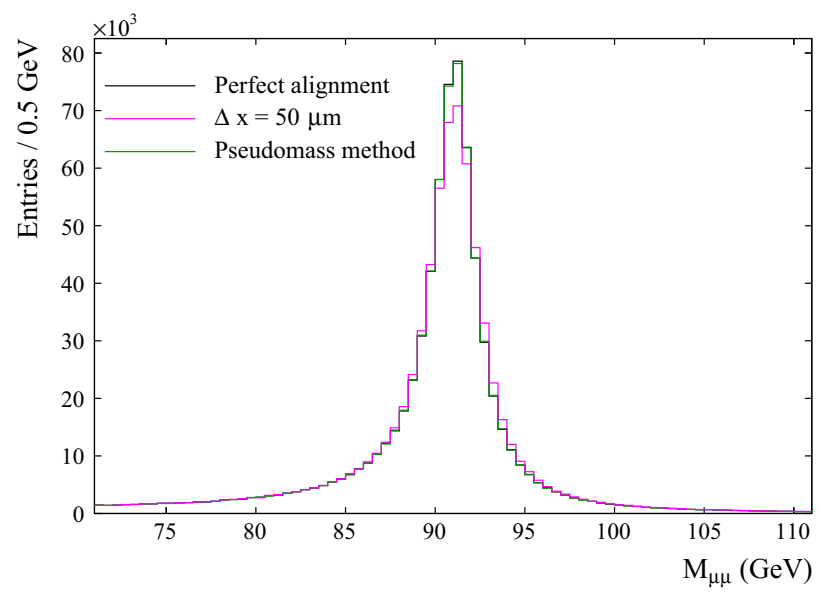

Fig. 6 The dimuon invariant mass distribution (left) and the forward backward asymmetry in bins of the dimuon invariant mass (right), for simulated events with a coherent $\Delta x=50 \mu \mathrm{m}$ (magenta), after applying

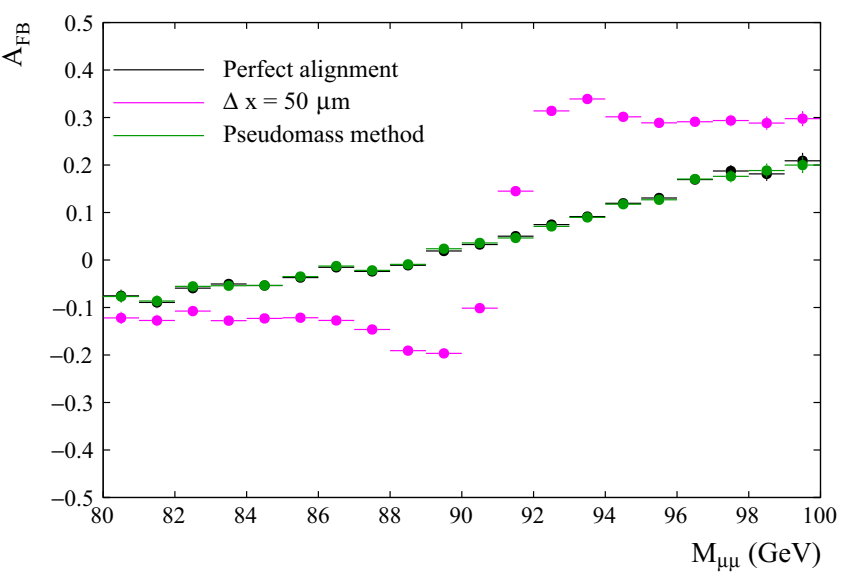

the pseudomass corrections (green), and the unbiased distribution, with no mis-alignment applied (black) 

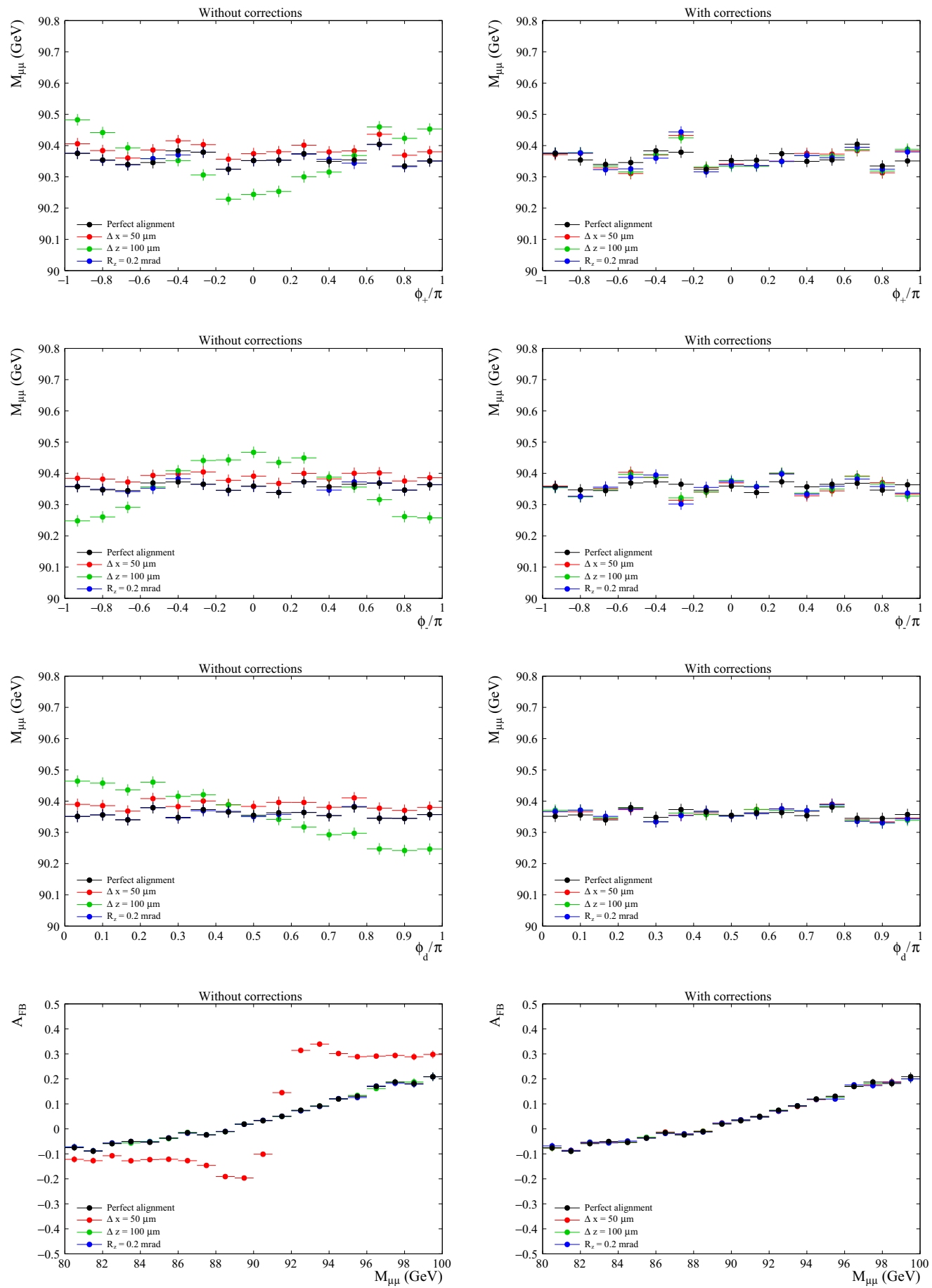

Fig. 7 The first three rows show the profiles of the mean dimuon invariant mass in bins of $\phi_{+}, \phi_{-}$and $\phi_{d}$. The lower row shows the $A_{F B}$ values in bins of mass. The black points correspond to the simulation with no mis-alignment while the other colours correspond to the first three

interest $\left(m_{W^{+}}, m_{W^{-}}\right.$and $\left.\sin ^{2} \theta_{\text {lept }}^{\text {eff }}\right) 90$ toy measurements are conducted.

The measurements of $m_{W}$ are based on template fits to the muon $p_{T}$ distribution of $W \rightarrow \mu \nu$ events, while the measurements of $\sin ^{2} \theta_{\text {lept }}^{\text {eff }}$ are based on template fits to $A_{F B}$ mis-alignment scenarios. The left and right columns correspond to the simulation before and after application of the pseudomass corrections, respectively

in bins of the dimuon mass in $Z \rightarrow \mu^{+} \mu^{-}$events. The data histograms are compared to templates where events are reweighted to emulate different $m_{W}$ and $\sin ^{2} \theta_{\text {lept }}^{\text {eff }}$ hypotheses. The toy experiments are configured differently for measurements of $m_{W}$ and $\sin ^{2} \theta_{\text {lept }}^{\text {eff }}$ : 

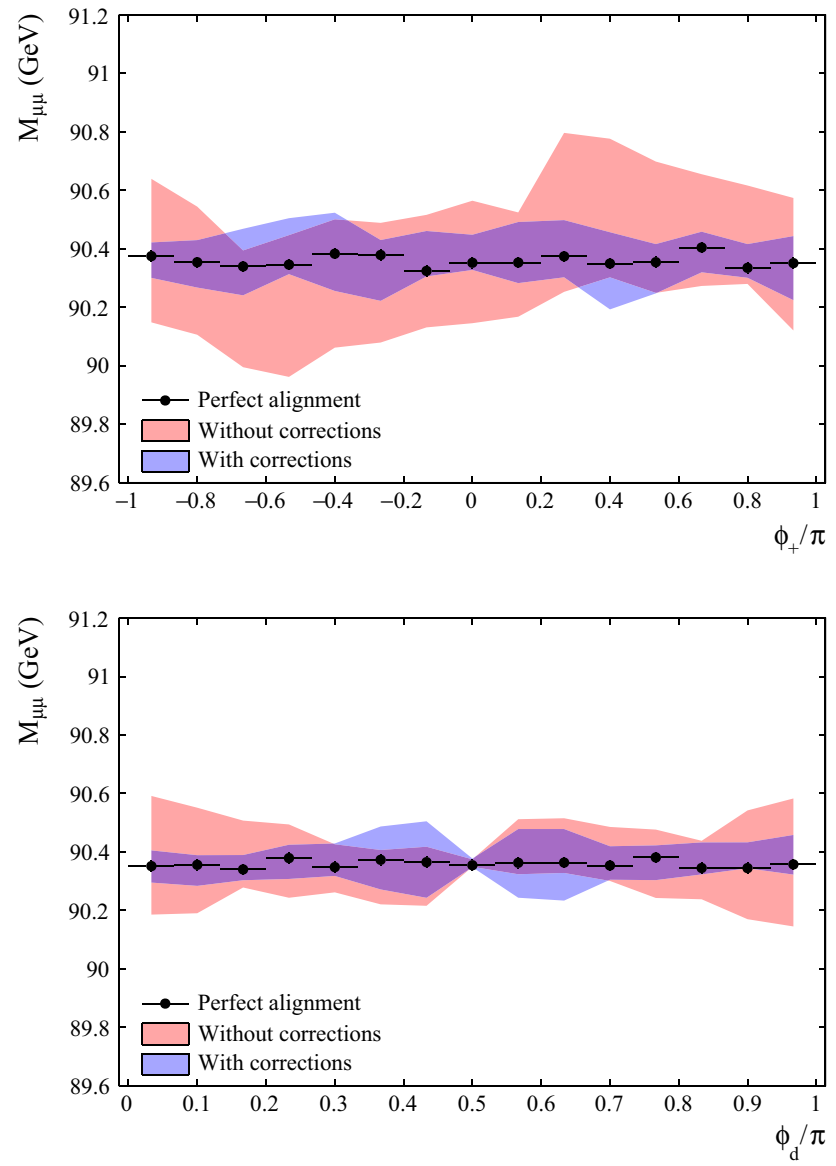

Fig. 8 Profiles of the mean dimuon invariant mass in bins of $\phi_{+}, \phi_{-}$ and $\phi_{d}$, and the $A_{F B}$ values in bins of mass. The black points correspond to the simulation before any mis-alignment. The red (blue) band represents the variations over ten toy experiments with the $100 \mu \mathrm{m}$ inco-
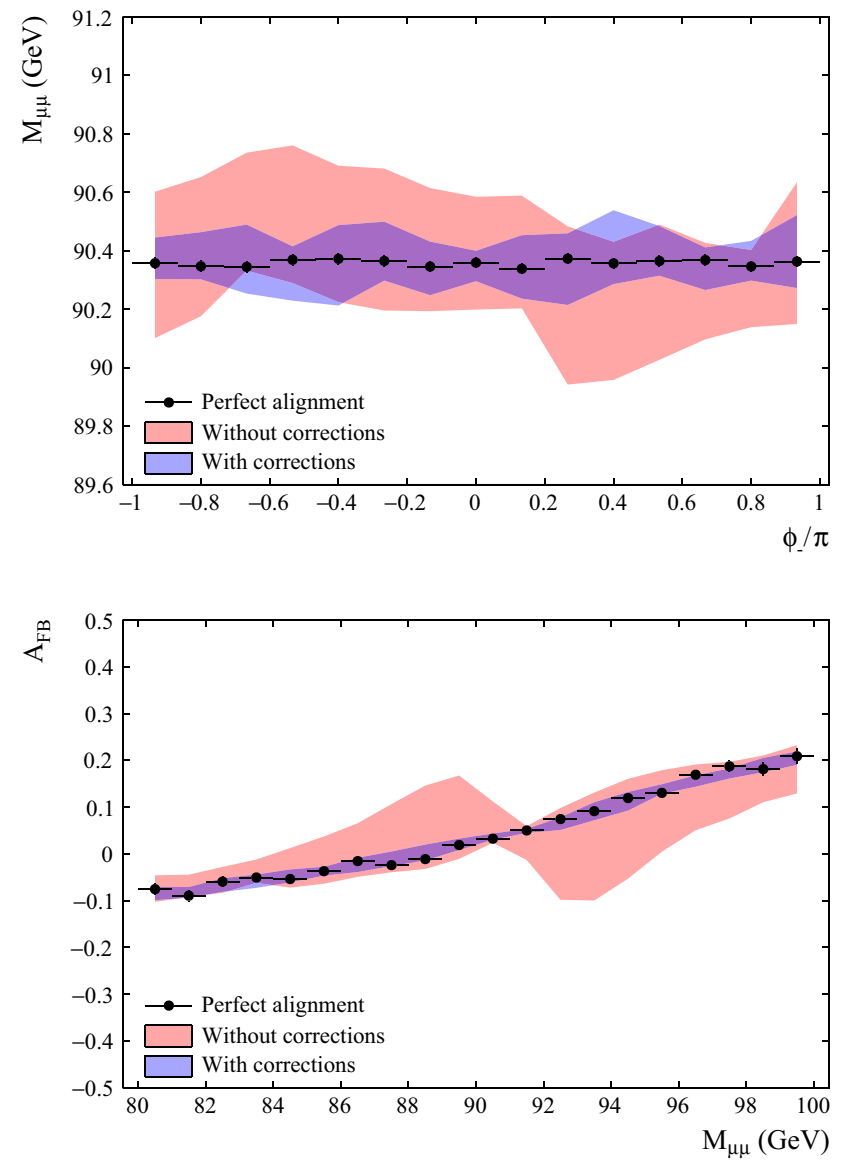

herent mis-alignment scenario before (after) application of the pseudomass method. The centre (width) of each band corresponds to the mean (RMS) of the ten toys

first four is-alignment scenarios in an example toy measurement. The overall bias in $m_{W}$, given by the weighted average of the biases for $m_{W^{+}}$and $m_{W^{-}}$, is also reported
Table 1 The biases in the values of $m_{W^{+}}, m_{W^{-}}$and $\sin ^{2} \theta_{\text {lept }}^{\text {eff }}$, and the correspondingshifts in the minimum $\chi^{2}$ values, that are caused by the

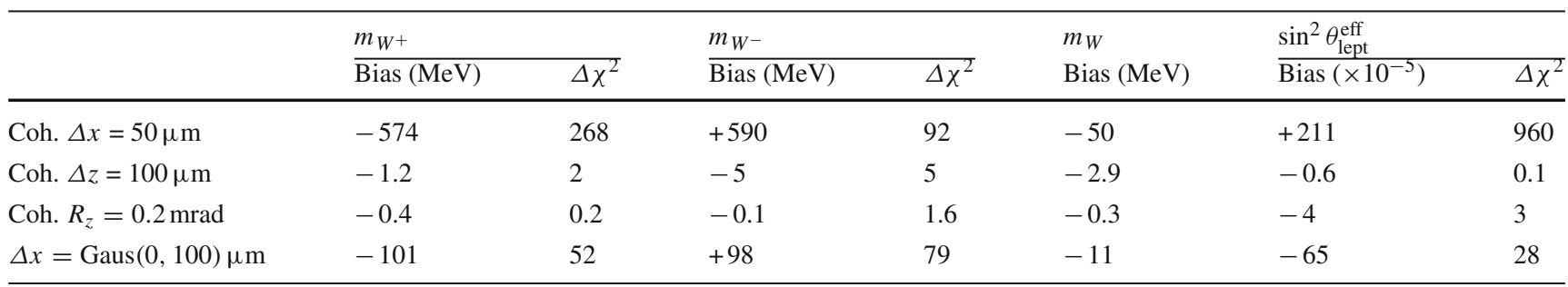

- In the case of $m_{W}$, toy data histograms are generated by randomly fluctuating the bins around the nominal muon $p_{T}$ distribution 90 times, assuming the expected $\mathrm{LHCb}$ Run-II yields [10] and Poisson statistics.

- The same procedure can not be used for the $A_{F B}$ templates, since the $Z \rightarrow \mu^{+} \mu^{-}$events used for the $\sin ^{2} \theta_{\text {lept }}^{\text {eff }}$ determination are also used to determine the pseudomass alignment corrections. It is therefore crucial to check whether this re-use of events causes any bias in the determination of $\sin ^{2} \theta_{\text {lept }}^{\text {eff }}$. Therefore, 90 statistically independent samples are selected from the original simulated data set.

For each data histogram a single-parameter fit determines the $m_{W}$ or $\sin ^{2} \theta_{\text {lept }}^{\text {eff }}$ value that minimises the $\chi^{2}$ between the data and the templates. The $68 \%$ C.L. statistical uncertainty 


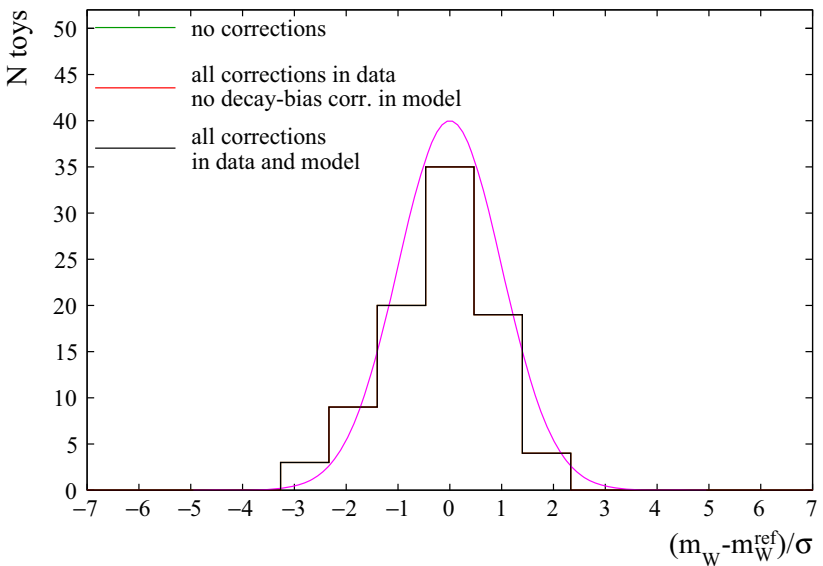

Fig. 9 The pull distributions for 90 toy measurements of (left) $m_{W^{+}}$ and (right) $\sin ^{2} \theta_{\text {lept }}^{\text {eff }}$. Three different scenarios are represented: (1) the pseudomass and decay-asymmetry bias corrections are not included in the toy data and template events (green); (2) the pseudomass corrections are applied to toy data and templates, but the decay-asymmetry

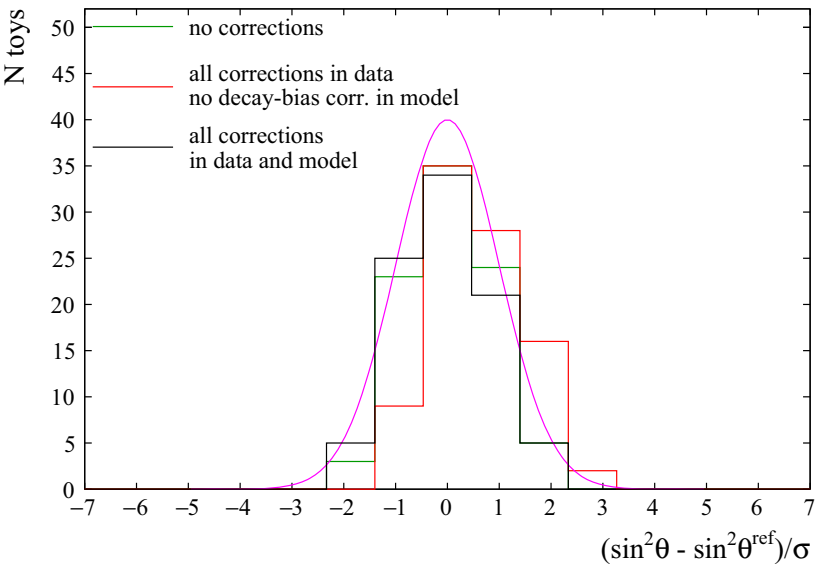

bias corrections are not included in the the templates (red); (3) the pseudomass and decay-asymmetry corrections are applied to both toy data and templates (black). Note that, in the case of $m_{W}$, the three distributions overlap. The expected statistical behaviour, represented by a unit Gaussian, is drawn in magenta

Table 2 The mean values of the distributions shown in red in Fig. 10 (where a $5 \mu \mathrm{m}$ mis-alignment along $x$ is applied to the toy data), before and after applying the pseudomass corrections. The biases on $m_{W}$ are reported in $\mathrm{MeV}$

\begin{tabular}{lllll}
\hline Bias in $m_{W^{+}}(\mathrm{MeV})$ & & Bias in $m_{W^{-}}(\mathrm{MeV})$ & & Bias in $\sin ^{2} \theta_{\text {lept }}^{\text {eff }}\left(\times 10^{-5}\right)$ \\
\hline Before (after) corr. & $\sigma_{\text {stat }}$ on $m_{W^{+}}$ & Before (after) corr. & $\sigma_{\text {stat }}$ on $m_{W^{-}}$ & Before (after) corr. \\
$-62(-1)$ & 9 & $+56(0.7)$ & 11 & $+26(9)$
\end{tabular}

corresponds to a variation of $\Delta \chi^{2}=1$ with respect to the parabola minimum.

The pull distributions for the extracted values of $m_{W^{+}}{ }^{3}$, and $\sin ^{2} \theta_{\text {lept }}^{\text {eff }}$ in 90 toys are shown in Fig. 9. What is denoted as "ref" value in the pull distributions is the nominal $m_{W}$ or $\sin ^{2} \theta_{\text {lept }}^{\text {eff }}$ value, which is aligned with the central template hypothesis. A Gaussian distribution with zero mean and unit width is drawn in magenta: this is the expected distribution over the 90 toys assuming reliable coverage of the statistical uncertainties. In order to study the impact of the pseudomass alignment method on the measurements of $m_{W}$ and $\sin ^{2} \theta_{\text {lept }}^{\text {eff }}$, before any mis-alignment, four different scenarios are considered.

1. No pseudomass correction is included in either the toy data or template events.

2. The pseudomass corrections are derived from the $Z$ sample and then applied to $W$ and $Z$ events when generating the data and template histograms. The curvature biases are determined with Eq. 5, i.e. without the small correction for the decay-asymmetry. This scenario is not shown in Fig. 9, but is discussed below.

\footnotetext{
${ }^{3}$ Qualitatively consistent results are seen for the $W^{-}$, so the figure is omitted for brevity.
}

3. Same as scenario 2, but with the decay-asymmetry bias corrections (Eq. 6) applied to only template events.

4. Same as scenario 3, but with the decay-asymmetry bias corrections applied to both toy data and template events.

It can be seen in Fig. 9 that in the first scenario both pull distributions are consistent with the ideal Gaussian functions. A small bias is observed in the $\sin ^{2} \theta_{\text {lept }}^{\text {eff }}$ pulls when the pseudomass corrections are applied without including the decay-asymmetry bias correction in the templates (red curve), thus introducing a discrepancy in the way the alignment corrections are applied to data and templates. However, the unbiased behaviour is restored after including the additional decay-asymmetry corrections. For better visualisation, the second scenario of the list above (no decay-asymmetry bias corrections in data and model) is not included in the plots, but an unbiased result is observed also in that case.

As a final test, it is interesting to check what happens to the four scenarios described above when a detector misalignment is included. Figure 10 shows the shift in the extracted $m_{W^{+}}$and $\sin ^{2} \theta_{\text {lept }}^{\text {eff }}$ values with respect to the reference value for the same 90 toys. The distributions drawn with blue lines are obtained from unbiased data. Those with red lines are obtained from data with a coherent $5 \mu \mathrm{m}$ misalignment along $x$. The upper (lower) row shows the results 

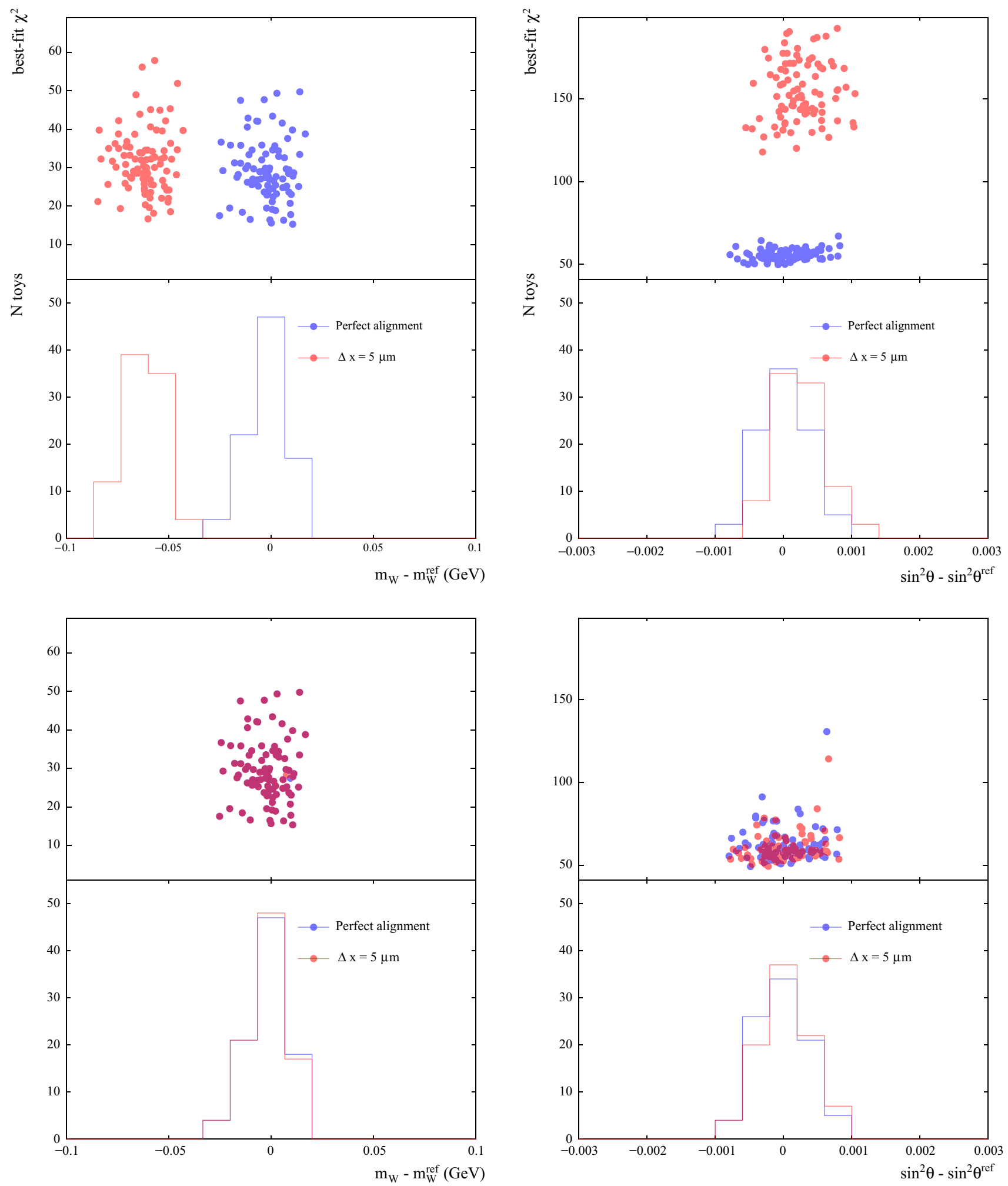

Fig. 10 The shift in the extracted (left) $m_{W^{+}}$and (right) $\sin ^{2} \theta_{\text {lept }}^{\text {eff }}$ values with respect to the reference value, in 90 toys. The upper (lower) row corresponds to the simulation before (after) application of the pseudomass method. The distributions drawn with blue lines are obtained from unbiased data. Those with red lines are obtained from toy data with a

coherent $5 \mu \mathrm{m}$ mis-alignment along $x$. The first (last) row of plots show the distributions before (after) applying the pseudomass corrections. The upper panel of each plot shows the best-fit $\chi^{2}$ corresponding to the extraction of $m_{W}$ and $\sin ^{2} \theta_{\text {lept }}^{\text {eff }}$ for the 90 toys 
before (after) applying the pseudomass corrections. In order to estimate the size of the bias on $m_{W}$ and $\sin ^{2} \theta_{\text {lept }}^{\text {eff }}$ driven by the introduced mis-alignment, the distributions in red are fitted with a Gaussian, and the corresponding means, before and after corrections, are reported in Table 2. A $60 \mathrm{MeV}$ bias in the extraction of $m_{W^{+}}$is estimated. Although the corresponding plot is not shown, we observe an opposite-sign bias of similar size for $m_{W^{-}}$. This confirms the sensitivity of the $m_{W}$ measurement to small mis-alignment effects. The same size mis-alignment has a smaller effect on the extraction of $\sin ^{2} \theta_{\text {lept }}^{\text {eff }}$, where the observed bias is within the statistical uncertainty of the measurement. However, the corrections have an impact in the best fit $\chi^{2}$ values extracted from each toy, as shown in the upper panel of each set of plots.

\section{Conclusion}

The measurements of, for example, $m_{W}$ and $\sin ^{2} \theta_{\text {lept }}^{\text {eff }}$, using muonic decays of weak bosons at hadron colliders are susceptible to biases in the measurement of muon momenta. A particular concern is curvature biases, caused by misalignments of the tracking detector elements, that depend on the sign of the particle charge. It is proposed to use the "pseudomass method", which is introduced in this paper, to determine corrections for charge-dependent curvature biases using $Z \rightarrow \mu^{+} \mu^{-}$decays. The method is validated using simulated $p p \rightarrow Z \rightarrow \mu^{+} \mu^{-}$events with the LHC RunII centre-of-mass energy of $13 \mathrm{TeV}$. A simplified model of a detector with a similar geometry to the LHCb experiment is used. This approach has the advantage of being less dependent on assumptions about the kinematics of the $Z$ boson decays than other methods present in the literature. A small correction for an effect of the forward-backward asymmetry in $Z \rightarrow \mu^{+} \mu^{-}$decays is required but, importantly, the curvature biases can be determined with limited sensitivity to assumptions about the value of $\sin ^{2} \theta_{\text {lept }}^{\text {eff }}$. The method is tested against several simplified mis-alignment configurations. With pseudo-experiments using simulated $Z \rightarrow \mu^{+} \mu^{-}$and $W \rightarrow \mu \nu$ decays it is demonstrated that the proposed method can be reliably applied in measurements of $m_{W}$ and $\sin ^{2} \theta_{\text {lept }}^{\text {eff }}$ in the presence of these simplified misalignments.

Acknowledgements We thank T. Wyatt for making the authors aware of the introduction, and usage of, the pseudomass variable in an analysis of data from the D0 experiment, which prompted the present study. We thank W. Hulsbergen for helpful suggestions that improve the clarity of our description of the pseudomass method and P. Ilten for his support with Monte Carlo generators. We also thank N. Tuning and L. Sestini for the useful comments, as well as O. Lupton, S. Farry, R. Hunter, M. Ramos Pernas, A. Chadwick, H. Yin and M. Xu for interesting discussions in the context of analyses of $W$ and $Z$ boson decays at LHCb. WB is supported by an Imperial College Research Fellowship. MV is supported by the grants ERC-CoG- 865469 SPEAR and STFCERF-ST/N004892/2.
Data Availability Statement This manuscript has no associated data or the data will not be deposited. [Authors' comment:The simulated data sample and the analysis framework used for this study can be accessed by anyone with LHCb user credentials, but they can be provided upon reasonable request.]

Open Access This article is licensed under a Creative Commons Attribution 4.0 International License, which permits use, sharing, adaptation, distribution and reproduction in any medium or format, as long as you give appropriate credit to the original author(s) and the source, provide a link to the Creative Commons licence, and indicate if changes were made. The images or other third party material in this article are included in the article's Creative Commons licence, unless indicated otherwise in a credit line to the material. If material is not included in the article's Creative Commons licence and your intended use is not permitted by statutory regulation or exceeds the permitted use, you will need to obtain permission directly from the copyright holder. To view a copy of this licence, visit http://creativecomm ons.org/licenses/by/4.0/.

Funded by $\mathrm{SCOAP}^{3}$.

\section{References}

1. M. Aaboud et al., Eur. Phys. J. C 78(2), 110 (2018). https://doi.org/ 10.1140/epjc/s10052-017-5475-4 [Erratum: Eur. Phys. J. C 78, 898 (2018)]

2. A. Bodek, A. van Dyne, J. Han, W. Sakumoto, A. Strelnikov, Eur. Phys. J. C 72, 2194 (2012). https://doi.org/10.1140/epjc/ s10052-012-2194-8

3. T.A. Aaltonen et al., Phys. Rev. D 89(7), 072005 (2014). https:// doi.org/10.1103/PhysRevD.89.072005

4. A.M. Sirunyan et al., Eur. Phys. J. C 78(9), 701 (2018). https://doi. org/10.1140/epjc/s10052-018-6148-7

5. J. Alves, A. Augusto et al., JINST 3, S08005 (2008). https://doi. org/10.1088/1748-0221/3/08/S08005

6. S. Alioli, P. Nason, C. Oleari, E. Re, JHEP 07, 060 (2008). https:// doi.org/10.1088/1126-6708/2008/07/060

7. P. Skands, S. Carrazza, J. Rojo, Eur. Phys. J. C 74(8), 3024 (2014). https://doi.org/10.1140/epjc/s10052-014-3024-y

8. T. Sjostrand, S. Mrenna, P.Z. Skands, JHEP 05, 026 (2006). https:// doi.org/10.1088/1126-6708/2006/05/026

9. G. Bozzi, L. Citelli, M. Vesterinen, A. Vicini, Eur. Phys. J. C 75(12), 601 (2015). https://doi.org/10.1140/epjc/s10052-015-3810-1

10. S. Farry, O. Lupton, M. Pili, M. Vesterinen, Eur. Phys. J. C 79(6), 497 (2019). https://doi.org/10.1140/epjc/s10052-019-6997-8

11. O. Lupton, M. Vesterinen, (2019). arXiv:1907.09958

12. R. Aaij et al., JHEP 11, 190 (2015). https://doi.org/10.1007/ JHEP11(2015)190

13. P. Azzi et al., CERN Yellow Rep. Monogr. 7, 1 (2019). https://doi. org/10.23731/CYRM-2019-007.1

14. V.M. Abazov et al., Phys. Rev. D 91(7), 072002 (2015). https://doi. org/10.1103/PhysRevD.91.072002

15. A. Banfi, S. Redford, M. Vesterinen, P. Waller, T.R. Wyatt, Eur. Phys. J. C 71, 1600 (2011). https://doi.org/10.1140/epjc/ s10052-011-1600-y. arXiv:1009.1580

16. T. Skwarnicki, A study of the radiative cascade transitions between the Upsilon-prime and Upsilon resonances. Ph.D. thesis, Institute of Nuclear Physics, Krakow (1986) (DESY-F31-86-02)

17. M.E.A. Tanabashi, Phys. Rev. D 98, 030001 (2018). https://doi. org/10.1103/PhysRevD.98.030001

18. R. Aaij et al., Int. J. Mod. Phys. A 30(07), 1530022 (2015). https:// doi.org/10.1142/S0217751X15300227 